\title{
Review: intensive blood pressure control and drugs reduce morbidity and mortality in hypertension and diabetes mellitus
}

\author{
Grossman E, Messerli FH, Goldbourt U. High blood pressure and diabetes mellitus. Are all antihypertensive drugs created \\ equal? Arch Intern Med 2000 Sep 11;160:2447-52.
}
QUESTION: In patients with hypertension and diabetes mellitus, what is the effectiveness of various antihypertensive treatments?

\section{Data sources}

Studies were identified by searching Medline (to June 1999) with the terms diabetes mellitus, hypertension, blood pressure, treatment, and therapy. Bibliographies of relevant studies were also checked.

\section{Study selection}

English language studies were selected if they were randomised controlled trials of $>12$ months duration that compared the effects of active drug treatments with placebo on morbidity and mortality in patients with hypertension and diabetes mellitus.

\section{Data extraction}

Data were extracted on patient characteristics, follow up period, blood pressure, rate of patients continuing to receive monotherapy, and cardiovascular morbidity and mortality.

\section{Main results}

8 studies (5244 patients) met the selection criteria. 3 studies compared 2 active treatments, 1 study reported the effects of intensive blood pressure lowering, and 3 studies compared active treatment with placebo. In 6 studies, mean ages ranged between 55 and 71 years; in 2 studies, patients were $\geqslant 60$ years of age. Follow up duration ranged between 2 and 8 years. Intensive blood pressure control reduced cardiovascular morbidity and mortality. One study showed that the calcium antagonist nisoldipine increased the incidence of fatal or non-fatal myocardial infarction more than did the angiotensin converting enzyme (ACE) inhibitor enalapril maleate $(11 \%$ v $2 \%$, relative risk increase $4 \%, 95 \%$ CI 1 to 11 , number needed to harm 12, CI 7 to 23). In the 3 studies that compared active treatment with placebo, a diuretic (chlorthalidone) and a calcium antagonist (nitrendipine) reduced cardiac end points, stroke, and total mortality. Comparisons between antihypertensive drugs were not possible because study populations differed. Except for nisoldipine, adverse events did not differ among drugs.

Sources of funding: various pharmaceutical companies.

For correspondence: Dr F H Messerli

Ochsner Clinic, 1514

Jefferson Highway, New

Orleans, LA 70121,

USA.Fax +1504842 4220 .

\section{Conclusion}

Intensive blood pressure control reduces cardiovascular morbidity and mortality in patients with hypertension and diabetes mellitus regardless of which of 4 classes of antihypertensive drugs (low dose diuretics, $\beta$ blockers, ACE inhibitors, and calcium antagonists) is used as first line treatment.

\section{COMMENTARY}

Achieving tight blood pressure control in patients with diabetes requires drug treatment, with most patients needing combination drug therapy. ${ }^{1}$ The choice of agent has been complicated by conflicting evidence of efficacy and safety. The review by Grossman $e t$ al shows that currently used agents are more effective than placebo for lowering cardiovascular risk in patients with diabetes and hypertension.

Does this mean that these agents are equivalent? Differences among agents are better assessed in head to head comparisons. The results of the review are consistent with a recently published meta-analysis that reviewed the same 4 randomised trials comparing ACE inhibitors with calcium antagonists, diuretics, and $\beta$ blockers. ${ }^{2}$ In 3 trials, ACE inhibitors lowered the risk for cardiovascular events (relative risk reduction [RRR] 51\%, CI 33 to 64 ) and all cause mortality more than did other agents (RRR 62\%, CI 13 to 62) with no effect on the risk for stroke. The fourth trial (the UK Prospective Diabetes Study) may not have had sufficient power to show differences between ACE inhibitors and $\beta$ blockers. ${ }^{3}$ These reports show that ACE inhibitors are similar to, if not better than, other antihypertensive drug classes for patients with diabetes.

Should ACE inhibitors be used as first line treatment in patients with diabetes and hypertension? Treatment with ramipril in patients with diabetes and hypertension lowers cardiovascular risk by $25 \%$ (CI 12 to 36$),{ }^{4}$ a reduction beyond its antihypertensive effect. This evidence supports the consideration of ACE inhibitors in all patients with diabetes and hypertension. Whatever the choice of agent, patients with diabetes and their clinicians should choose tight blood pressure control.

\section{Victor M Montori, MD} Mayo Clinic

Rochester, Minnesota, USA

1 UK Prospective Diabetes Study Group. Tight blood pressure control and risk of macrovascular and microvascular complications in type 2 diabetes: UKPDS 38. BMJ 1998;317:703-13.

2 Pahor M, Psaty BM, Alderman MH, et al. Therapeutic benefits of ACE inhibitors and other antihypertensive drugs in patients with type 2 diabetes. Diabetes Care 2000;23:888-92.

3 UK Prospective Diabetes Study Group. Efficacy of atenolol and captopril in reducing risk of macrovascular and microvascular complications in type 2 diabetes: UKPDS 39. BMJ 1998:317:713-20.

4 Heart Outcomes Prevention Evaluation Study Investigators. Effects of ramipril on cardiovascular and microvascular outcomes in people with diabetes mellitus: results of the HOPE study and MICRO-HOPE substudy. Lancet 2000;355:253-9. 